AIRWAY BIOLOGY

\title{
Retinoic acid fails to reverse emphysema in adult mouse models
}

\author{
M Fujita, Q Ye, H Ouchi, N Nakashima, N Hamada, N Hagimoto, K Kuwano, R J Mason, \\ Y Nakanishi
}

See end of article for authors' affiliations

.....................

Correspondence to: M Fujita, MD, Research Institute for Disease of the Chest, Graduate School of Medical Sciences, Kyushu University, 3-1-1,

Maidashi, Higashiku, Fukuoka 812-8582, Japan; fujita@kokyu.med. kyushu-u.ac.jp

Received 27 May 2003 Accepted 6 December 2003
Background: Previous work has shown that all-trans-retinoic acid reverses elastase induced emphysema in rats. Since there is currently no effective treatment for pulmonary emphysema, the effect of retinoic acid should be further investigated in other adult species. A study was undertaken using two murine models of emphysema to evaluate the effect of retinoic acid.

Methods: The models used were an elastase induced emphysema model for acute alveolar destruction and a tumour necrosis factor (TNF)- $\alpha$ transgenic mouse which exhibits chronic air space enlargement, loss of elastic recoil, increased lung volume, and pulmonary hypertension comparable to human pulmonary emphysema. All-trans-retinoic acid $(2 \mathrm{mg} / \mathrm{kg}$ ) was injected for 12 successive days after the establishment of emphysema. The effects of treatment were evaluated using physiological and morphometric analyses. Results: In contrast to the rat, administration of all-trans-retinoic acid in these murine models did not improve the emphysema. Moreover, worsening of emphysema was observed in TNF- $\alpha$ transgenic mice treated with all-trans-retinoic acid. The level of keratinocyte chemoattractant (KC), a CXC chemokine, in bronchoalveolar lavage fluid was increased in TNF- $\alpha$ transgenic mice following retinoic acid treatment. These data raise the possibility that retinoic acid causes deterioration of emphysema by promoting inflammation in this model.

Conclusions: In these models, retinoic acid did not show positive effects on emphysema. The effect of retinoic acid in the treatment of pulmonary emphysema remains controversial, and further studies are required to determine its physiological effects under a variety of experimental conditions.
$\mathrm{P}$ ulmonary emphysema is a serious disease characterised by reduced maximal expiratory flow, increased lung volume, and alveolar wall destruction. ${ }^{1}$ There is a strong relationship with tobacco smoking, and as many as 2 million people in the United States suffer from pulmonary emphysema. Furthermore, the prevalence seems to be increasing. Although there has been extensive research into the molecular mechanisms underlying the development of emphysema, the clinical management of patients with pulmonary emphysema remains mostly supportive. There is no proven clinically effective treatment to promote recovery from established emphysema. ${ }^{23}$ Recently, all-trans-retinoic acid has been reported to reverse elastase induced emphysema in rats. ${ }^{4}$ Administration of all-trans-retinoic acid, a metabolite of retinol (vitamin A), to rats with elastase induced emphysema attenuated alveolar destruction and increased the alveolar number, resulting in normal alveolar morphometry. Although retinoic acid had a positive effect when given to mice aged less than 2 months, ${ }^{5}$ it has not been found to have a beneficial effect on the recovery from emphysema in any adult animal model other than the rat. In addition, a recent clinical trial of all-trans-retinoic acid in the treatment of chronic obstructive pulmonary disease (COPD) did not result in any improvement. ${ }^{6}$

The aim of this study was to determine the effect of retinoic acid on pulmonary emphysema in mice using two murine models of air space enlargement-namely, treatment with elastase ${ }^{4}$ and surfactant protein (SP)-C/tumour necrosis factor (TNF) $\alpha$ transgenic mice. The transgenic mouse constitutionally overexpresses TNF- $\alpha$ under the SP-C promoter and develops air space enlargement, loss of elastic recoil, increased lung volumes, and ultimately pulmonary hypertension. ${ }^{78}$ This model is particularly appropriate since
TNF- $\alpha$ is considered to be an important factor in the development of pulmonary emphysema. ${ }^{9}$

\section{METHODS}

\section{Animals}

Eight week old C57BL/6 female mice were used in the study. Porcine pancreatic elastase was purchased from Elastin Product Co (Owensville, MI, USA). After anaesthesia with bromoethanol, a 22 gauge feeding tube with a tip bent to 45 degrees was inserted into the trachea and advanced into the left bronchus. Three units of elastase in $50 \mu \mathrm{l}$ saline were injected into the left lung.

SP-C/TNF- $\alpha$ transgenic mice bred into the C57BL/6 background were also used. Transgenic male mice were crossed with normal C57BL/6 female mice and bred in an animal facility documented to be free of murine specific pathogens. Mice were genotyped and transgenic negative littermates were used as controls. Female mice aged 24-28 weeks were studied because emphysematous change is complete at this age. $^{7}$

The study was approved by the Institutional Animal Care and Use Committee of our institute.

\section{Retinoic acid administration}

All-trans-retinoic acid was dissolved in cottonseed oil in the dark. Retinoic acid $(2 \mathrm{mg} / \mathrm{kg})$ in $100 \mu \mathrm{l}$ volume was injected intraperitoneally for 12 successive days after the development of pulmonary emphysema. Control mice were injected with cottonseed oil.

Abbreviations: Cstat, static expiratory compliance; FRC, functional residual capacity; KC, keratinocyte chemoattractant; TLC, total lung capacity; TNF- $\alpha$, tumour necrosis factor $\alpha$ 
Table 1 Number of mice, body weights, and lung volumes in elastase model and in TNF$\alpha$ transgenic mice used in this experiment

\begin{tabular}{lllll}
\hline & Control & & Elastase \\
\hline Treatment & OIL & RA & OIL & RA \\
$n$ & 5 & 5 & 5 & 5 \\
Body weight (g) & $21.7(0.02)$ & $20.8(0.03)$ & $21.2(0.02)$ & $22.5(0.03)$ \\
& & & & \\
& Littermates & & Transgenic & RA \\
Treatment & OIL & RA & OIL & 6 \\
$N$ & 6 & 6 & 6 & $22.8(0.69)$ \\
Body weight (g) before & $23.4(1.03)$ & $23.3(1.03)$ & $22.6(0.29)$ & $23.8(0.64)$ \\
Body weight (g) after & $24.3(1.28)$ & $23.9(0.98)$ & $23.8(0.80)$ & $0.98(0.07)$ \\
Lung volume (ml) & $0.43(0.01)$ & $0.42(0.02)$ & $0.96(0.07)$ & \\
\hline Oll = cottonseed oil; RA = retinoic acid. & & & \\
Values in parentheses are standard errors. & & & \\
\hline
\end{tabular}
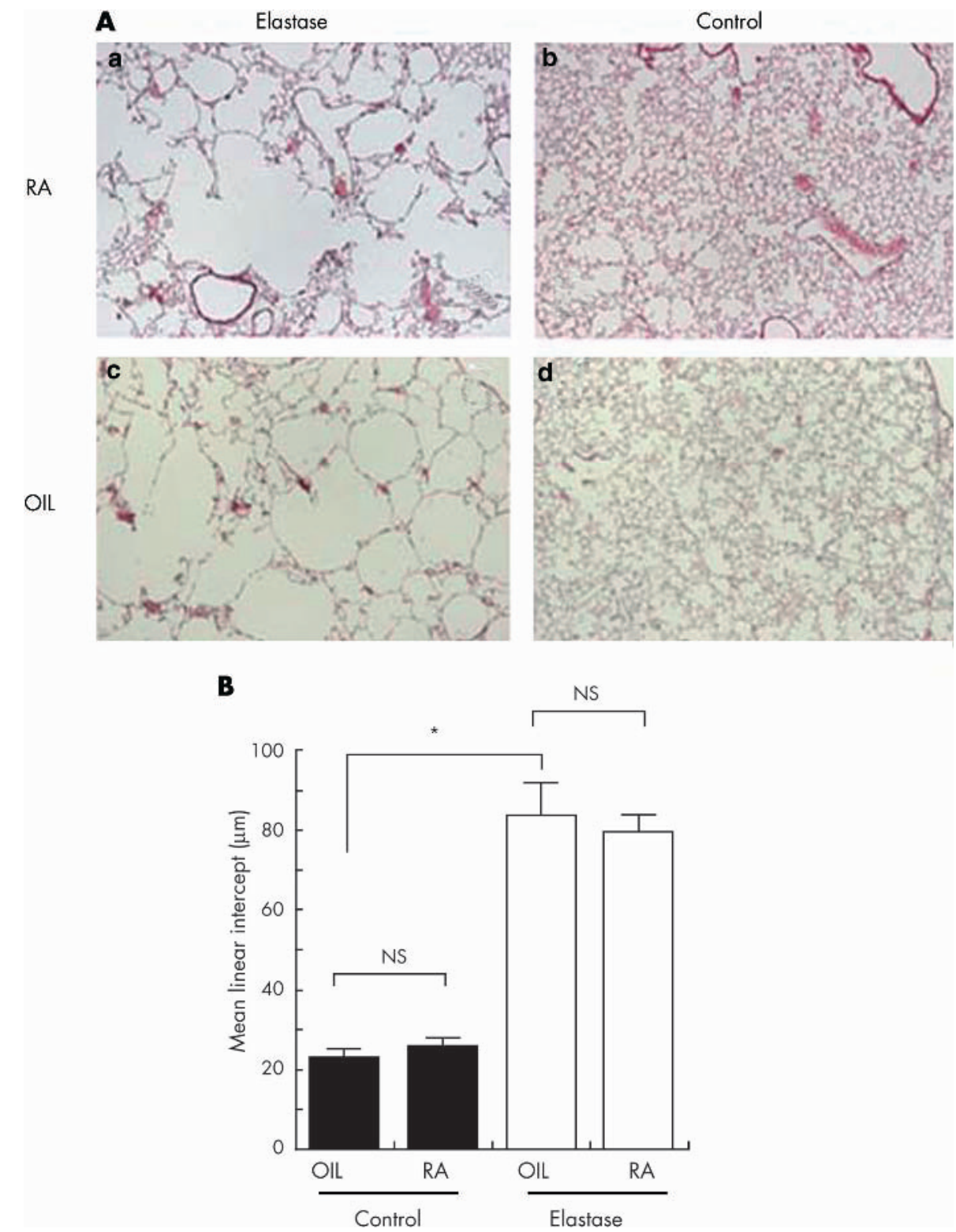

Figure 1 Histology and morphometric analysis of lungs from mice with elastase induced emphysema and controls. (A) Representative photomicrographs showing lung morphology in the four experimental groups: (a) elastase injected mice treated with retinoic acid (RA), (b) saline injected mice treated with RA, (c) elastase injected mice treated with cottonseed oil (OIL), and (d) saline injected mice treated with OIL. There were no apparent differences between treatment with RA or OIL. All slides were stained with haematoxylin/eosin. Original magnification $\times 40$. (B) Morphometric analyses of lungs from control and elastase injected mice treated with either OIL or RA. No difference was evident between control mice treated with OIL or RA. Elastase treatment resulted in a marked increase in the mean linear intercept (Lm), but there was no significant difference between mice treated with either OIL or RA $(p=0.43$, mean difference $-0.25,95 \% \mathrm{Cl}-1.77$ to 1.27$) .{ }^{*} p<0.0001$ elastase treated $v$ controls. 

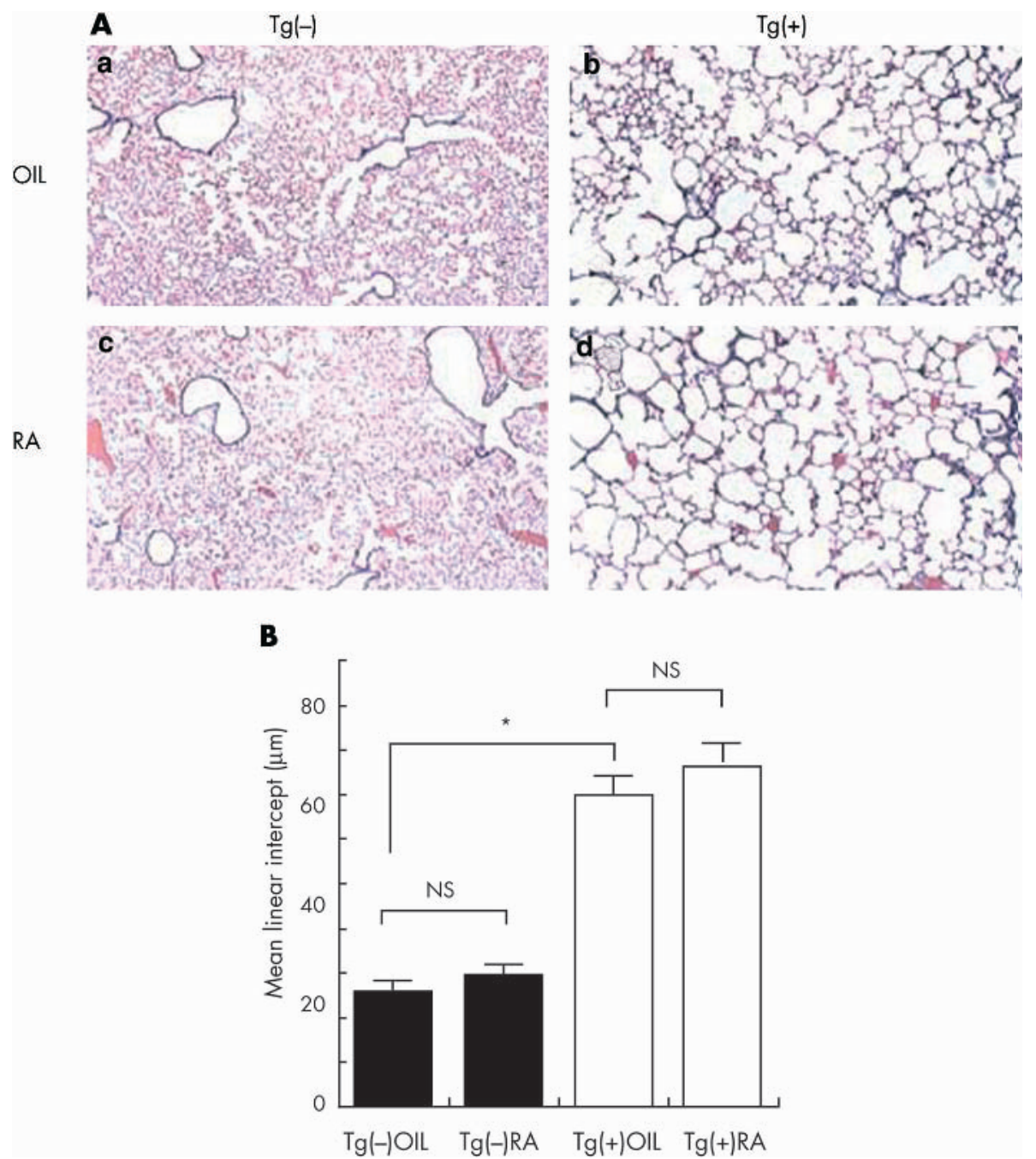

Figure 2 Histology and morphometric analysis of lungs from SP-C/TNF- $\alpha$ transgenic mice and controls. (A) Representative photomicrographs showing lung morphology in the four experimental groups: (a) control mice $\mathrm{Tg}(-)$ treated with cottonseed oil (OIL), (b) $\mathrm{Tg}(-)$ mice treated with retinoic $\operatorname{acid}(\mathrm{RA})$, (c) SP-C/TNF- $\alpha$ transgenic mice Tg(+) treated with OIL, (d) Tg(+) mice treated with RA. There were no significant differences between RA and OIL treatments. All slides were stained with haematoxylin/eosin. Original magnification $\times 40$. (B) Morphometric analyses of lungs from TNF- $\alpha$

transgenic and control mice treated with either OIL or RA. No difference was evident between control $\mathrm{Tg}(-)$ mice treated with either OIL or RA. Tg(+) mice developed a marked increase in Lm but no significant difference was evident between Tg(+) mice treated with either OIL or RA ( $p=0.14$; mean difference $0.71,95 \% \mathrm{Cl}-0.56$ to 1.99). ${ }^{*} \mathrm{p}<0.0001 \mathrm{TNF}-\alpha$ transgenic mice $v$ transgene negative littermates $(\mathrm{n}=6$ in each group).

Pulmonary physiology, histology, and morphometry After 12 days of treatment with retinoic acid or cottonseed oil, a number of pulmonary physiological parameters were examined using previously described methods. ${ }^{7}$ The mice were then killed by intraperitoneal injection of sodium pentobarbital. Lungs were inflated at $25 \mathrm{~cm} \mathrm{H}_{2} \mathrm{O}$ static pressure by intratracheal instillation of $4 \%$ paraformaldehyde in phosphate buffered saline (PBS). Tissue sections were stained with haematoxylin and eosin and morphometric studies were performed as described previously. ${ }^{70}$ The mean linear intercept $(\mathrm{Lm})$, an indicator of air space size, was calculated for each mouse from 10 randomly selected fields at a magnification of $\times 200$ using a 21 line counting grid.

\section{Bronchalveolar lavage (BAL) studies}

After insertion of a tracheal tube, the trachea was lavaged five times with $1 \mathrm{ml}$ aliquots of PBS. Cells in the lavage fluid were counted with a haemocytometer. Differential cell counts of BAL fluid were performed on 200 cells from a smear stained with a modified Wright's stain (DiffQuik; American Scientific Products, McGas Park, IL, USA). For gelatin zymography, $40 \mu \mathrm{l}$ of BAL fluid was electrophoresed in a sodium dodecyl sulfate-polyacrylamide (SDS) gel containing $1 \%$ gelatin under non-reducing conditions as described previously. ${ }^{7}$ The level of keratinocyte chemoattractant (KC) in the BAL fluid was measured by enzyme linked immunosorbent assay (ELISA) according to the manufacturer's instructions (Genzyme Techne, Minneapolis, MN, USA).

\section{Statistical analysis}

Data are expressed as mean (SE) values. The statistical analyses were performed using SAS software, Version 6. Analysis of variance (ANOVA) was performed, using the Tukey-Kramer test to adjust for multiple comparisons. A p value of $<0.05$ was considered to indicate a significant difference.

\section{RESULTS}

\section{Retinoic acid toxicity}

In a preliminary experiment the same dose of retinoic acid $(500 \mu \mathrm{g} / \mathrm{kg})$ as was used to treat elastase induced emphysema in rats $^{4}$ was given to four TNF- $\alpha$ transgenic mice and their 


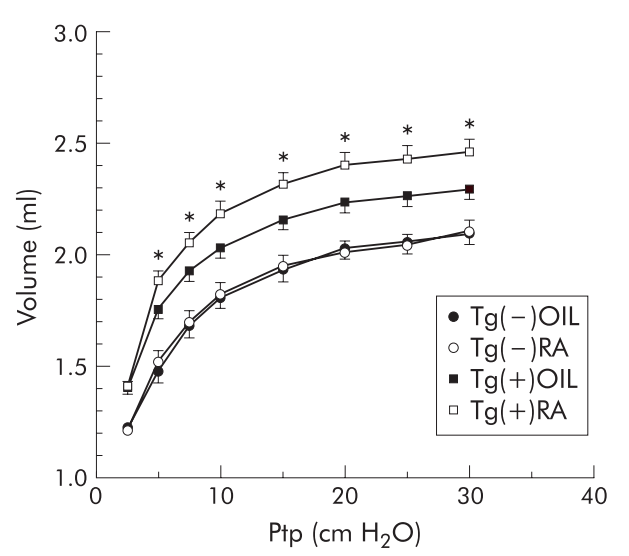

Figure 3 Expiratory phase of pressure-volume curves from TNF- $\alpha$ transgenic and control mice $(\mathrm{Tg}(+)$ and $\mathrm{Tg}(-)$, respectively) treated with either cottonseed oil (OIL) or retinoic acid (RA). Each group consisted of six mice. Asterisk indicates statistical difference between RA and OIL treatments in TNF- $\alpha$ transgenic mice. There was no difference between RA and OIL treatments in Tg(-) littermate controls.

littermates. No significant histological or physiological differences were found between the retinoic acid and cottonseed oil groups. Moreover, retinoic acid given in a dose of $100 \mu \mathrm{g} / \mathrm{kg}$ also resulted in no significant differences in the pathophysiology in TNF- $\alpha$ transgenic mice and their littermates ( $\mathrm{n}=3$ each). As a result, the dose of retinoic acid was increased to $2 \mathrm{mg} / \mathrm{kg}$. Retinoic acid administration did not produce noticeable morbidity and mortality. Although Meshi et $a l^{11}$ reported excess deaths in guinea pigs treated
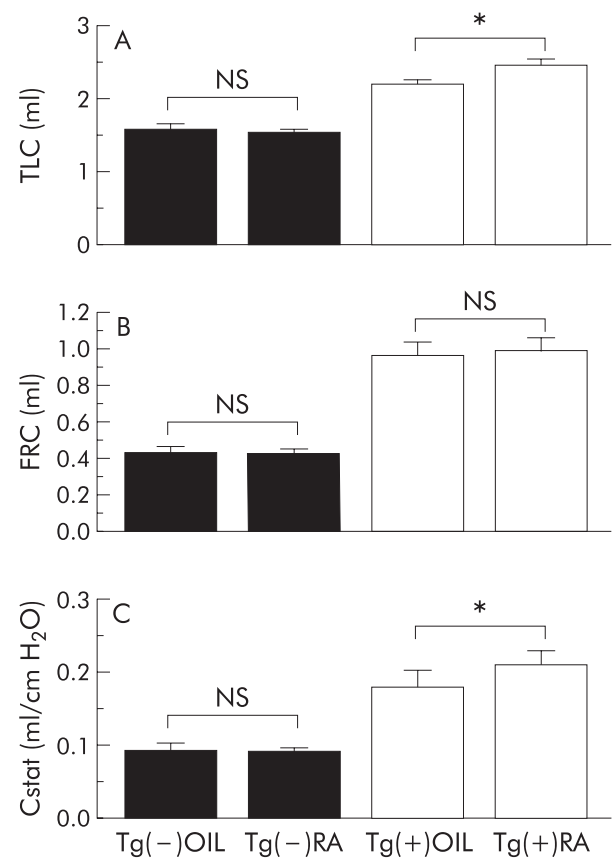

Figure 4 (A) Total lung capacity (TLC), (B) functional residual capacity (FRC) and (C) static compliance (Cstat) in TNF- $\alpha$ transgenic (Tg(+)) and transgene negative littermates $(\operatorname{Tg}(-))$ treated with either cottonseed oil (OIL) or retinoic acid (RA). Each group consisted of six mice. TLC was significantly increased in $\mathrm{Tg}(+)$ mice treated with RA compared with $\mathrm{Tg}(+)$ mice treated with OIL. Asterisk indicates statistical difference between RA and OIL treatments (TLC: mean difference $-0.28195 \% \mathrm{Cl}$ -0.60 to 0.04$), p=0.03$; Cstat; mean difference $-0.01(95 \% \mathrm{Cl}-0.03$ to 0.01 ), $p=0.04$ ). There was no difference between RA and OIL treatments in control mice. with retinoic acid, no mice died in this experiment. Body weights were measured during the administration of cottonseed oil and retinoic acid and there was no difference between the control and experimental groups. The number of mice, body weight, and lung volumes are summarised in table 1 .

Effect of retinoic acid on elastase induced emphysema Elastase administration into the left lung induced severe haemorrhagic pneumonia on day 1 . There was progressive alveolar destruction which resulted in emphysema and air space enlargement 28 days later. Intraperitoneal administration of retinoic acid was started 28 days after elastase administration and continued for 12 successive days. Histopathological examination indicated no obvious difference between retinoic acid treated mice and controls (fig lA), and this was confirmed by morphometric analysis (fig 1B). Elastase treatment produced a marked increase in Lm which was not reduced by retinoic acid. Administration of cottonseed oil did not induce any structural pulmonary changes compared with administration of saline (fig lA).

\section{Effect of retinoic acid in TNF- $\alpha$ transgenic mice}

SP-C/TNF- $\alpha$ transgenic mice developed alveolar enlargement, increased lung volumes, and chronic inflammation at 6 months of age, as previously reported. ${ }^{7}$ Retinoic acid did not affect the gross histology of the lungs of TNF- $\alpha$ transgenic mice (fig 2A). The presence of small alveoli, previously reported in elastase induced emphysema in rats following retinoic acid treatment, were not observed in TNF- $\alpha$ transgenic mice treated with retinoic acid. Morphometric analysis indicated a tendency for retinoic acid to induce alveolar enlargement in TNF- $\alpha$ transgenic mice although this was not statistically significant (fig $2 \mathrm{~B}$ ). Six transgenic mice and six littermates were each treated with either cottonseed oil or retinoic acid.

\section{Pulmonary physiology}

The pressure-volume (PV) relationships in the various experimental groups are shown in fig 3. Administration of retinoic acid resulted in a left shift of the PV curve in TNF- $\alpha$ transgenic mice, indicating the development of more severe and progressive emphysematous changes, but there was no change in the PV relationship in the littermates. Total lung capacity (TLC) and static expiratory compliance (Cstat) were increased in TNF- $\alpha$ transgenic mice following retinoic acid administration, but functional residual capacity (FRC) was unchanged (fig 4). These data indicate that retinoic acid administration induced a deterioration in emphysema-like changes in TNF- $\alpha$ transgenic mice. In the murine elastase induced emphysema model the effect of treatment was evaluated by morphometry since the disease is localised to the left lung in this model.

\section{Analyses of cells counts, metalloproteinases and chemokine levels in BAL fluid}

BAL fluid was collected from both retinoic acid and cottonseed oil treated mice in both models and cell counts performed. Four mice in each group were used for this experiment. Treatment of mice with elastase induced emphysema with retinoic acid did not result in any significant change in the differential cell count of BAL fluid (data not shown). Neutrophils were increased in BAL fluid from TNF- $\alpha$ transgenic mice before retinoic acid treatment as previously described. ${ }^{7}$ However, no difference was apparent between transgenic mice treated with retinoic acid or cottonseed oil (fig 5).

Matrix metalloproteinases (MMPs) have been reported to account for the development of COPD. ${ }^{12}$ Indeed, BAL fluid 


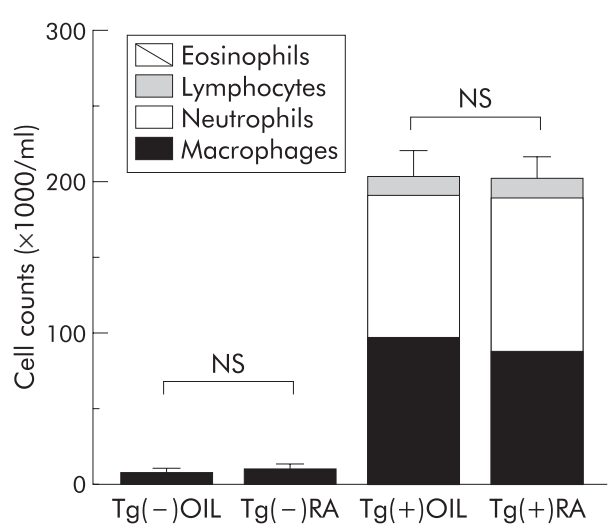

Figure 5 Cell counts in bronchoalveolar lavage (BAL) fluid in TNF- $\alpha$ transgenic $(\operatorname{Tg}(+))$ and control mice $(\operatorname{Tg}(-))$ treated with retinoic acid (RA) or cottonseed oil (OIL). Each group consisted of four mice. There was no significant difference between RA and OIL treatments.

from TNF- $\alpha$ transgenic mice exhibited MMP activity, mainly gelatinase. ${ }^{7}$ BAL fluid from mice treated with retinoic acid or cottonseed oil was examined by gelatin zymography but no difference in gelatinase activity was observed between the two groups in either the elastase induced emphysema model or in TNF- $\alpha$ transgenic mice (data not shown).

$\mathrm{KC}$ is a CXC chemokine which plays an important role in neutrophil accumulation while interleukin 8 (IL-8), a human CXC chemokine, has been reported to contribute to the development of pulmonary emphysema. ${ }^{13}$ Since retinoic acid has been reported to induce IL-8, the concentration of KC in BAL fluid from retinoic acid treated mice was measured by ELISA. The BAL fluid concentration of KC was significantly increased until $\mathrm{l}$ day after the administration of elastase and was undetectable at 28 days (fig 6A). In wild type mice no significant difference was observed in the BAL fluid concentration of KC between groups treated with retinoic acid or cottonseed oil. In fact the BAL fluid concentration of KC level was significantly higher in retinoic acid treated TNF- $\alpha$ transgenic mice than in TNF- $\alpha$ transgenic mice treated with cottonseed oil (fig 6B). In addition, TNF- $\alpha$ transgenic mice treated with cottonseed oil had higher levels of $\mathrm{KC}$ than transgene negative littermates.

\section{DISCUSSION}

Emphysema is regarded as a progressive disease related to cigarette smoking, with no effective treatment after emphysematous changes have been established. A report that retinoic acid can restore the normal lung structure in rats with established elastase induced emphysema ${ }^{4}$ was therefore of considerable interest. In addition, a small study reported a reduced concentration of vitamin $\mathrm{A}$ in patients with moderate to severe COPD with an improvement in pulmonary function testing being achieved after vitamin A supplementation. $^{14}$ These data suggested that retinoic acid administration may represent a new treatment for human emphysema. However, in a recent clinical trial of the effect of all-trans-retinoic acid in patients with COPD, no significant improvement was achieved. ${ }^{6}$ In addition, retinoic acid did not promote recovery from smoking induced emphysema in the guinea pig. ${ }^{11}$ These studies suggest that the beneficial effect of retinoic acid treatment may be species specific. We therefore examined the effect of retinoic acid in murine models of emphysema.

In the murine elastase induced emphysema model we evaluated the effect of treatment by morphometry alone since disease is localised to the left lung in this model. No significant histological changes were seen following retinoic acid treatment, and we therefore conclude that it is ineffective in promoting recovery in the elastase model of emphysema in mice. This finding is consistent with the study recently reported by Lucey et $a l^{15}$ in which retinoic acid was started at day 22 after elastase administration. We speculate that retinoic acid does not affect alveolar septation in the murine model.

TNF- $\alpha$ transgenic mice have increased lung volumes, loss of elastic recoil, destruction of alveolar walls, and pulmonary hypertension. ${ }^{78}$ Since these changes progress with age, chronic inflammation induced by TNF- $\alpha$ plays an important role in developing emphysema. These mice appeared to show emphysematous changes rather than pulmonary fibrosis as originally reported. ${ }^{16}$ In these mice retinoic acid also failed to reverse the emphysema as defined by both morphometric and physiological parameters. It had no effect on the morphometric measurement of alveolar size but appeared to have a tendency to cause deterioration of emphysematous changes, although the difference was not statistically significant. Physiological assessment showed an increase in TLC, static compliance, and a left shift in the PV curve. These physiological data indicate a deterioration in pulmonary emphysema in TNF- $\alpha$ transgenic mice after retinoic acid
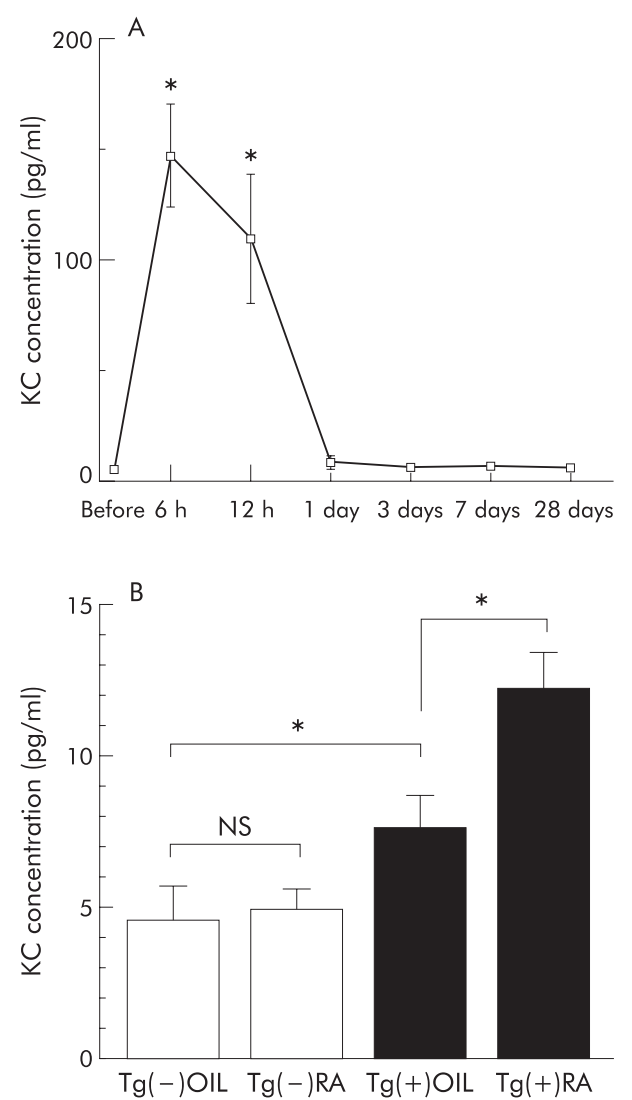

Figure 6 Concentration of keratinocyte chemoattractant $(\mathrm{KC})$ in $\mathrm{BAL}$ fluid. (A) Kinetics of $K C$ concentration in BAL fluid after administration of elastase. The KC concentration was markedly increased at 6 and 12 hours after elastase administration and fell thereafter. Each group consisted of four mice. Asterisk indicates statistical difference compared with the KC concentration before treatment $(6$ hours; $p<0.0001$,

12 hours; $p=0.0001)$. (B) KC concentration in TNF- $\alpha$ transgenic $(\mathrm{Tg}(+))$ and control $(\mathrm{Tg}(-))$ mice treated with retinoic acid (RA) and cottonseed oil (OIL). Each group consisted of four mice. Asterisk indicates statistical difference between RA and OIL treatments in TNF- $\alpha$ transgenic mice $(p=0.01$, mean difference $-4.65,95 \% \mathrm{Cl}-8.60$ to -0.69$)$ and also between TNF- $\alpha$ transgenic mice and Tg(-) littermates $(p=0.05$, mean difference $-2.67,95 \% \mathrm{Cl}-8.35$ to 3.00 ). 
treatment. Since the finding of increased lung volume and decreased elastic recoil following treatment with retinoic acid in TNF- $\alpha$ transgenic mice was unexpected, the mechanism underlying the deterioration in lung function induced by retinoic acid was investigated further.

Vitamin A is known to have a critical role in the differentiation, growth, and health of lung epithelial cells. Vitamin A deficiency results in a loss of mucus secreting epithelium because cells are unable to differentiate normally and develop as layers of keratinising squamous epithelium. ${ }^{17}$ This finding led to a clinical trial to establish if vitamin $\mathrm{A}$ supplementation might prevent lung cancer, but vitamin A and $\beta$ carotene both caused an increase in the incidence of lung cancer compared with a control group. ${ }^{18}$ The reason for this adverse result remains unclear, although some researchers have reported that vitamin A might induce inflammation which may outweigh its beneficial antioxidant effects. ${ }^{19-21}$ Retinoic acid may induce DNA damage. ${ }^{20}$ Moreover, Harant et al reported that simultaneous treatment with retinoic acid and TNF- $\alpha$ resulted in an increase in the IL- 8 mRNA level in vitro. ${ }^{19}$ This induction is likely to result from the interaction of retinoic acid with the NF- $\mathrm{KB}$ site and IL-8 promoter. ${ }^{21}$ In addition, retinoic acid upregulateed TNF receptors and potentiated TNF induced activation of $N F-\kappa B$, activated protein 1, and apoptosis. ${ }^{22}$ This is of interest since the human CXC chemokine IL-8 is associated with the development of pulmonary emphysema. Neutrophil infiltration induced by IL-8 causes alveolar destruction through neutrophil elastase and MMP activation. ${ }^{13}$ In the present study we found that the concentration of the CXC chemokine KC was increased early in the course of elastase induced emphysema in mice. In addition, a higher level of KC was found in the BAL fluid of $\mathrm{TNF}-\alpha$ transgenic mice with emphysema than in control littermates. We therefore consider that KC actively contributes, at least in part, to the development of emphysema in mice. TNF- $\alpha$ transgenic mice treated with retinoic acid had a higher KC level than controls. The increase in KC after retinoic acid was only seen in the TNF- $\alpha$ transgenic mice. Although there was a tendency to increased neutrophil accumulation in TNF- $\alpha$ transgenic mice treated with retinoic acid, it was not statistically significant. A similar finding has been observed in humans. The level of IL- 8 in BAL fluid was correlated with neutrophil elastase $/ \alpha_{1}$-protease complex inhibitor but not with neutrophil numbers. ${ }^{13}$ It is probably difficult to show a significant increase in neutrophil accumulation by $\mathrm{KC}$ in TNF- $\alpha$ transgenic mice since these mice already have high levels of neutrophils in their BAL fluid. An alternative explanation might be the increase in $\mathrm{KC}$ stimulated neutrophil function in TNF- $\alpha$ transgenic model treated with retinoic acid.

On the other hand, retinoic acid has been reported to reduce intrapulmonary inflammation, ${ }^{23}{ }^{24}$ but we do not know the precise reason for the difference. Further studies are needed to clarify this controversy.

These data indicate that retinoic acid does not induce regeneration of alveoli in mice with established emphysema. In contrast to the elastase induced model of pulmonary emphysema, TNF- $\alpha$ transgenic mice exhibit persistent inflammation during the period of retinoic acid administration. Pulmonary emphysema was therefore exacerbated by retinoic acid. This suggests that the presence of severe inflammation might be a risk factor for worsening of alveolar injury following retinoic acid treatment. Moreover, treatment with retinoic acid in a guinea pig model of smoking induced emphysema resulted in increased deaths. ${ }^{11}$ In view of the fact that current thinking suggests an important central role for inflammation in the pathogenesis of COPD, care should be taken when considering the use of all-trans-retinoic acid as a potential therapeutic agent in patients with COPD, especially during the early phase of the disease.

It is difficult to know why retinoic acid has different effects in rats and mice since lung retinoid signalling is highly conserved. ${ }^{25-29}$ It is also difficult to interpret the different effects of retinoic acid in different models of emphysema such as elastase or overexpression of TNF- $\alpha$. It is possible that the effect of retinoic acid is specific to the model, especially inflammation. TNF- $\alpha$ transgenic mice still had inflammation when retinoic acid was administered, as shown by BAL fluid analysis. Another possible explanation is the age of the animals. In this study we used 8 week old mice for the elastase model and 24-28 week old transgenic mice. Even in the same background $(\mathrm{C} 57 \mathrm{Bl} / 6)$, the effect of retinoic acid on emphysema was different. Previous studies have used 4 month old rats in the elastase induced emphysema model and reported recovery from emphysema. ${ }^{4}{ }^{15}$ It has previously been shown that retinoic acid affected the emphysema model when administered to mice less than 2 months of age. ${ }^{5}$ In older mice the ability to generate alveoli may be lost and administration of retinoic acid may also result in inflammation. Another possibility is the dose of retinoic acid used. We used a dose of retinoic acid that was four times larger than the dose used in rats. ${ }^{4}$ Other unknown mechanisms for controlling alveoli generation may exist and should be investigated in further studies.

In summary, retinoic acid may exert disparate effects in emphysema with either resolution or exacerbation depending on the dose used, the age and species of animal, and the degree of underlying inflammation. The dose and duration of administration of retinoic acid should therefore be considered carefully in clinical trials. Further study is required to determine the effect of retinoic acid on emphysema.

\section{Authors' affiliations}

M Fujita, Q Ye, H Ouchi, N Nakashima, N Hamada, N Hagimoto, K Kuwano, Y Nakanishi, Research Institute for Diseases of the Chest, Graduate School of Medical Sciences, Kyushu University, Fukuoka, Japan

R J Mason, Department of Medicine, National Jewish Medical and Research Center, Denver, CO, USA

The study was supported in part by a grant (14570553) from Scientific Research from the Ministry of Education, Science and Culture of Japan (to MF).

\section{REFERENCES}

1 Pauwels R, Buist A, Calverley $P$, et al. Global strategy for the diagnosis, management, and prevention of chronic obstructive pulmonary disease. NHLBI/WHO global initiative for chronic obstructive lung disease (GOLD) workshop summary. Am J Respir Crit Care Med 2001;163:1256-76.

2 Hogg JC. A stimulating treatment for emphysema. Nature Med 1997;3:603-5.

3 Utz JP, Hubmayr RD, Deschamps C. Lung volume reduction surgery for emphysema: out on a limb without a NETT. Mayo Clin Proc 1998;73:552-66.

4 Massaro GDC, Massaro D. Retinoic acid treatment abrogates elastaseinduced pulmonary emphysema in rats. Nature Med 1997;3:675-7.

5 Massaro GD, Massaro D. Retinoic acid treatment partially rescues failed septation in rats and in mice. Am J Physiol Lung Cell Mol Physiol 2000;278:L955-60.

6 Mao JT, Goldin JG, Dermand J, et al. A pilot study of all-trans-retinoic acid for the treatment of human emphysema. Am J Respir Crit Care Med 2002; 165:718-23.

7 Fujita M, Shannon JM, Irvin CG, et al. Overexpression of tumor necrosis factor- $\alpha$ produces an increase in lung volumes and pulmonary hypertension. Am J Physiol Lung Cell Mol Physiol 2001;280:L39-49.

8 Fujita M, Mason RJ, Cool C, et al. Pulmonary hypertension in TNF-alpha overexpressing mice is associated with decreased VEGF gene expression. $J$ Appl Physiol 2002;93:2162-70.

9 Churg A, Dai J, Tai $\mathrm{H}$, et al. Tumor necrosis factor-alpha is central to acute cigarette smoke-induced inflammation and connective tissue breakdown. Am J Respir Crit Care Med 2002;166:849-54.

10 Dunnill MS. Quantitative methods in the study of pulmonary pathology. Thorax 1962; 17:320-8.

11 Meshi B, Vitalis TZ, lonescu D, et al. Emphysematous lung destruction by cigarette smoke. The effects of latent adenoviral infection on the lung inflammatory response. Am J Respir Cell Mol Biol 2000;26:52-7. 
12 Hautamaki RD, Kobayashi DK, Senior RM, et al. Requirement for macrophage elastase for cigarette smoke-induced emphysema in mice. Science 1997;277:2002-4

13 Tanino M, Betsuyaku T, Takeyabu K, et al. Increased levels of interleukin-8 in BAL fluid from smokers susceptible to pulmonary emphysema. Thorax 2002;57:405-11

14 Paiva SA, Godoy I, Vannucchi H, et al. Assessment of vitamin A status in chronic obstructive pulmonary disease patients and healthy smokers. Am J Clin Nutr 1996;64:928-34.

15 Lucey EC, Goldstein RH, Breuer R, et al. Retinoic acid does not affect alveolar septation in adult FVB mice with elastase-induced emphysema. Respiration 2003;70:200-5

16 Miyazaki Y, Araki K, Vesin C, et al. Expression of a tumor necrosis factor-alpha transgene in murine lung causes lymphocytic and fibrosing alveolitis. A mouse model of progressive pulmonary fibrosis. J Clin Invest 1995;96:250-9.

17 Chytil F. The lungs and vitamin A. Am J Physiol 1992;262:L517-27.

18 Omenn GS, Goodman GE, Thornquist MD, et al. Risk factors for lung cancer and for intervention effects in CARET, the beta-carotene and reinol efficacy trial. J Natl Cancer Inst 1996;88:1550-9.

19 Harant H, Martin Rd, Andrew PJ, et al. Synergistic activation of interleukin-8 gene transcription by all-trans-retinoic acid and tumor necrosis factor-alpha involves the transcription factor NF-kappaB. J Biol Chem 1996;271:26954-61.

20 Murata M, Kawanishi S. Oxidative DNA damage by vitamin A and its derivative via superoxide generation. J Biol Chem 2000;275:2003-8.
21 Chang MMJ, Harper R, Hyde DM, et al. A novel mechanism of retinoic acidenhanced interleukin-8 gene expression in arway epithelium. Am J Respir Cell Mol Biol 2000;22:502-10.

22 Manna SK, Aggarwal BB. All-trans-retinoic acid upregulates TNF receptors and potentiates TNF-induced activation of nuclear factors-kappaB, activated protein-1 and apoptosis in human lung cancer cells. Oncogene 2000; 19:2110-9.

23 Paquette NC, Zhang LY, Ellis WA, et al. Vitamin A deficiency enhances ozone-induced lung injury. Am J Physiol 1996;270:L475-82.

24 Massaro D, Massaro GDC. Retinoids, alveolus formation, and alveolar deficiency. Clinical implications. Am J Respir Cell Mol Biol 2003;28:271-4.

25 Ong DE, Chytil F. Changes in levels of cellular retinol- and retinoic-acidbinding proteins of liver and lung during perinatal development of rat. Proc Natl Acad Sci USA 1976;73:3976-8.

26 McGowan S, Jackson SK, Jenkins-Moore M, et al. Mice bearing deletions of retinoic acid receptors demonstrate reduced lung elastin and alveolar numbers. Am J Respir Cell Mol Biol 2000;23:162-7.

27 Massaro GDC, Massaro D, Chan W-Y, et al. Retinoic acid receptor-b: an endogenous inhibitor of the perinatal formation of pulmonary alveoli. Physiol Genomics 2000;4:51-7.

28 Hind $M$, Corcoran J, Maden M. Alveolar proliferation, retinoid synthesizing enzymes, and endogenous retinoids in the postnatal mouse lung. Different roles for aldh-1 and raldh-2. Am J Respir Cell Mol Biol 2002;26:67-73.

29 Hind M, Corcoran J, Maden M. Pre- and postnatal lung development, maturation, and plasticity. Temporal/spatial expression of retinoid binding proteins and RAR isoforms in the postnatal lung. Am J Physiol Lung Cell Mol Physiol 2002;282:L468-76.

\section{LUNG ALERT}

\section{Does CT screening for lung cancer assist smokers to stop?}

$\Delta$ Cox LS, Clark MM, Jett JR, et al. Change in smoking status after spiral chest computed tomography scan screening Cancer 2003;98:2495-501

$\mathrm{S}$ moking cessation is of paramount importance in the prevention of lung cancer. Attendance for cancer screening may provide a "teachable moment". This study aimed to evaluate changes in smoking status following lung cancer screening in a large group of both current and ex-smokers. 1520 subjects were enrolled and underwent low dose spiral chest CT scan screening for lung cancer. $97 \%$ returned at 1 year follow up for assessment of smoking status (confirmed in $48 \%$ by exhaled CO measurement) and were included in the analysis. Baseline characteristics predictive of abstinence from smoking were identified using logistic regression. Variables studied included age, sex, average number of cigarettes per day, number of years smoked, $\mathrm{FEV}_{1}$ (\% predicted), results of the baseline screening test and, for ex-smokers, duration of abstinence at baseline.

$14 \%$ of the 901 baseline current smokers were abstinent at follow up, higher than the expected rate of $5-7 \%$ in a general population. After adjusting for other potential variables, lower $\mathrm{FEV}_{1} \%$ predicted was the only significant predictor of abstinence from smoking at 1 year. $10 \%$ of the 574 baseline ex-smokers had resumed smoking, similar to rates seen in smoking cessation programmes. A longer duration of abstinence at baseline was the only significant predictor of continued smoking abstinence.

Since the abstinence rate was higher than in a general population and a negative screening test did not affect the quit rate, the authors conclude that screening is an opportunity to provide additional assistance in smoking cessation and to implement relapse prevention measures in former smokers. However, since the study did not include a comparison group of subjects without CT scan screening, a direct evaluation of the role of this intervention in changing smoking status is not possible.

$\mathrm{N}$ Jayasekera

SHO, London Chest Hospital, London, UK nivenkajayasekera@hotmail.com 